\title{
Can High-Dose Eicosapentaenoic Acid Get a Place as a Plaque Modifier?
}

\author{
Kota Murai, MD; Yu Kataoka, MD, PhD; Teruo Noguchi, MD, PhD
}

D espite the established anti-atherosclerotic benefits of lowering low-density lipoprotein-cholesterol (LDL-C) with a statin, atherosclerotic coronary artery disease still remains one of the leading causes of death in developed countries, which underscores the need to modify additional residual risks. The n-3 fatty acids (eicosapentaenoic acid (EPA) or docosahexaenoic acid (DHA)) have been considered as a potential therapeutic target to prevent atherosclerotic cardiovascular diseases. Although the JELIS study reported a significant reduction in cardiovascular events with EPA in Japanese subjects, ${ }^{1} 2$ recent randomized controlled trials (RCTs: REDUSE-IT study $^{2}$ and STRENGTH study ${ }^{3}$ ) have reported inconsistent findings about the clinical benefits of agents modulating EPA and/or DHA in the secondary prevention setting under statin therapy. In addition, a recent cohort study reported that these contrasting results were driven partly by differences of comparator oils. ${ }^{4}$ These observations indicate further need to conduct dedicated studies for elucidating the efficacy of targeting EPA and/or DHA.

In this issue of the Journal, Motoyama et al ${ }^{5}$ used serial coronary computed tomography angiography (CCTA) to compare atheroma progression in 210 acute coronary syndrome (ACS) statin-treated subjects receiving different doses of EPA/DHA. The main findings of their study are: (1) types of statin differed in the 4 groups, and a lower ontreatment LDL-C level was observed in those treated with high-dose EPA+DHA or high-dose EPA alone, (2) on serial CCTA imaging analysis, the frequency of plaque progression was $0 \%$ and $7.1 \%$ in the high-dose EPA+DHA and high-dose EPA alone groups, respectively, (3) when patients were stratified into 3 groups according to the dose of EPA, less plaque progression was observed in the highdose EPA group, (4) the dose of DHA was not associated with the degree of plaque progression, and (5) the use of high-dose EPA more likely induced reduction of fibrous, fibrofatty and low-attenuation plaque volumes with less calcification.

Although this study provides additional clinical data to support the favorable anti-atherosclerotic effect of highdose EPA, several limitations should be considered when interpreting the findings. First, this was a single-center, observational study, not a randomized controlled clinical trial. The type and dose of statin and the use of EPA/DHA

\section{Article p????}

agents were decided at each physician's discretion not by randomization. As a consequence, significant differences exist in the type of statin and on-treatment LDL-C among the 4 groups. In particular, given that patients receiving high-dose EPA+DHA and high-dose EPA exhibited a lower on-treatment LDL-C level, this lipid management may slow their plaque progression rate. Second, it is important to evaluate the efficacy of therapies under guideline-recommended LDL-C control, whereas the on-treatment LDL-C level in the current study subjects was $>70 \mathrm{mg} / \mathrm{dL}$. Whether high-dose EPA still works even in ACS patients who achieve on-treatment LDL-C $<70 \mathrm{mg} / \mathrm{dL}$ remains unknown. Third, calcification is considered as a contributor to plaque stabilization, but in the high-dose EPA and high-EPA groups, calcification was not promoted. Further investigation is required into whether high-dose EPA truly stabilizes coronary atherosclerosis after ACS. Further studies are required to clarify whether high-dose EPA favorably modifies plaque quality and quantity in statin-treated ACS patients with optimal LDL-C control.

To date, several RCTs have used a variety of plaque imaging modalities to investigate the efficacy of n-3 fatty acids on coronary atherosclerosis ${ }^{6-14}$ (Table). Although 6 studies reported a reduction of plaque volume or a plaque stabilization effect in those receiving EPA, others did not show any positive findings. In addition, subanalysis of the STRENGTH study reported that achieving a greater EPA level with the agent did not necessarily reduce cardiovascular events. ${ }^{15}$ These inconsistent observations indicate the complicated properties of EPA and DHA in vivo. Further elucidation of their physiological and biological effects in circulation is needed to determine whether "high-dose" EPA truly modulates coronary atherosclerosis under statin therapy.

Disclosures

T.N. is a member of Circulation Journal's Editorial Team.

\section{IRB Information}

Not applicable.

The opinions expressed in this article are not necessarily those of the editors or of the Japanese Circulation Society.

Received November 17, 2021; accepted November 18, 2021; J-STAGE Advance Publication released online December 17, 2021

Department of Cardiovascular Medicine, National Cerebral and Cardiovascular Center, Suita, Japan

Mailing address: Kota Murai, MD, Department of Cardiovascular Medicine, National Cerebral and Cardiovascular Center, 6-1 Kishibe-shimmachi, Suita 564-8565, Japan. E-mail: murai.kota11@ncvc.go.jp

All rights are reserved to the Japanese Circulation Society. For permissions, please e-mail: cj@j-circ.or.jp

ISSN-1346-9843 


\begin{tabular}{|c|c|c|c|c|c|}
\hline \multirow{2}{*}{$\begin{array}{l}\text { Imaging } \\
\text { modality } \\
\text { Study }\end{array}$} & \multicolumn{2}{|c|}{$\begin{array}{c}\text { Coronary computed tomography } \\
\text { angiography (CСТA) }\end{array}$} & \multicolumn{3}{|c|}{ Intravascular ultrasound (IVUS) } \\
\hline & Alfaddagh et al ${ }^{6}$ (2017) & Budoff et al7 (2020) & Niki et al8 (2016) & Ahn et al ${ }^{9}(2016)$ & Watanabe et al ${ }^{10}(2017)$ \\
\hline Subjects & Stable CAD & $\begin{array}{l}\text { Patients with coronary } \\
\text { artery stenosis on } \\
\text { CCTA, with elevated } \\
\text { triglycerides }\end{array}$ & Stable CAD & Stable CAD and ACS & Stable CAD and ACS \\
\hline Therapies & $\begin{array}{c}126: 114 \text { for EPA } \\
1.86 \mathrm{~g} / \text { day }+ \text { DHA } \\
1.5 \mathrm{~g} / \text { day vs. no } \\
\mathrm{n}-3 \text { fatty acids }\end{array}$ & $\begin{array}{c}\text { 31:37 for EPA } \\
4 \mathrm{~g} / \text { day vs. placebo }\end{array}$ & $\begin{array}{c}29: 30 \text { for EPA } \\
1.8 \mathrm{~g} / \text { day vs. no } \\
\text { n-3 fatty acids }\end{array}$ & $\begin{array}{c}38: 36 \text { for EPA } \\
1.395 \mathrm{~g} / \text { day + DHA } \\
1.125 \mathrm{mg} / \text { day vs. placebo }\end{array}$ & $\begin{array}{l}97: 96 \text { for pitavastatin } \\
4 \mathrm{mg} / \text { day }+ \text { EPA } \\
1.8 \mathrm{mg} / \text { day vs. } \\
\text { pitavastatin } 4 \mathrm{mg} / \text { day }\end{array}$ \\
\hline $\begin{array}{l}\text { Percentage of } \\
\text { statin-treated } \\
\text { patients }\end{array}$ & $95 \%$ & $100 \%$ & $100 \%$ & $100 \%$ & $47 \%$ \\
\hline $\begin{array}{l}\text { Baseline LDL-C } \\
\text { level in each } \\
\text { group, mg/dL }\end{array}$ & $\begin{array}{c}78.5 \text { vs. } 77.5 \\
(P=0.46)\end{array}$ & $\begin{array}{l}\text { Not shown but not } \\
\text { significantly different } \\
\text { between groups }\end{array}$ & $\begin{array}{l}97.7 \text { vs. } 100.0 \\
\quad(P=0.85)\end{array}$ & $\begin{array}{c}127.0 \text { vs. } 113.8 \\
\quad(P=0.100)\end{array}$ & $\begin{array}{c}107.1 \text { vs. } 98.6 \\
(P=0.080)\end{array}$ \\
\hline Type of statins & $\begin{array}{l}\text { Both high- and } \\
\text { low-intensity statins }\end{array}$ & Not shown & $\begin{array}{l}\text { Atorvastatin or } \\
\text { rosuvastatin or pitavastatin }\end{array}$ & $\begin{array}{l}\text { Atorvastatin or } \\
\text { rosuvastatin }\end{array}$ & Pitavastatin \\
\hline $\begin{array}{l}\text { On-treatment } \\
\text { LDL-C in each } \\
\text { group, mg/dL }\end{array}$ & $\begin{array}{l}84.6 \text { vs. } 80.4 \\
\quad(P=0.76)\end{array}$ & $\begin{array}{l}\text { Not shown but increase } \\
\text { not significantly different } \\
\text { between groups }\end{array}$ & $\begin{array}{c}91.4 \text { vs. } 88.3 \\
\text { (Not evaluated) }\end{array}$ & $\begin{array}{c}86.8 \text { vs } 80.8 \\
(P=0.525)\end{array}$ & $\begin{array}{c}76.9 \text { vs } 76.0 \\
(P=0.796)\end{array}$ \\
\hline $\begin{array}{l}\text { Primary } \\
\text { outcome }\end{array}$ & $\begin{array}{l}\text { Change in noncalcified } \\
\text { plaque volume at } \\
30 \text { months }\end{array}$ & $\begin{array}{l}\text { Change in } \\
\text { low-attenuation plaque } \\
\text { volume at } 18 \text { months }\end{array}$ & $\begin{array}{l}\text { Changes in plaque } \\
\text { components assessed } \\
\text { by integrated } \\
\text { backscatter-IVUS } \\
\text { at } 6 \text { months }\end{array}$ & $\begin{array}{l}\text { Changes in atheroma } \\
\text { volume index and } \\
\text { percent atheroma } \\
\text { volume at } 12 \text { months }\end{array}$ & $\begin{array}{c}\text { Coronary plaque volume } \\
\text { and composition } \\
\text { assessed by integrated } \\
\text { backscatter-IVUS at } 6-8 \\
\text { months }\end{array}$ \\
\hline Findings & $\begin{array}{c}\text { Primary endpoint not } \\
\text { significantly different } \\
\text { between groups } \\
(-2.4 \% \text { vs. } 4.5 \% \\
P=0.14)\end{array}$ & $\begin{array}{l}\text { EPA group reduced } \\
\text { low-attenuation plaque } \\
\text { volume }(-17 \% \text { vs. } \\
+109 \%, P=0.0061)\end{array}$ & $\begin{array}{l}\text { EPA group reduced } \\
\text { lipid plaque volume } \\
(-18.9 \% \text { vs. }+8.4 \% \text {, } \\
\text { P=0.002) and increased } \\
\text { fibrous volume }(+11.7 \% \\
\text { vs. }-9.2 \%, P=0.01)\end{array}$ & $\begin{array}{c}\text { Primary endpoint not } \\
\text { significantly different } \\
\text { between groups (atheroma } \\
\text { volume index: }-12.65 \% \text { vs. } \\
-8.51 \%, P=0.768 \text { and } \\
\text { percent atheroma volume: } \\
-4.36 \% \text { vs. }-9.98 \%, P=0.526 \text { ) }\end{array}$ & $\begin{array}{c}\text { EPA group reduced total } \\
\text { atheroma volume } \\
\left(-9.3 \mathrm{~mm}^{3} \text { vs. }-1.7 \mathrm{~mm}^{3} \text {, }\right. \\
\mathrm{P}<0.001) \text {. Lipid volume } \\
\text { decreased in EPA group } \\
\text { only }(-3.4 \%, \mathrm{P}=0.045 \text { vs. } \\
-1.3 \%, P=0.429)\end{array}$ \\
\hline
\end{tabular}

\begin{tabular}{|c|c|c|c|c|}
\hline \multirow{2}{*}{$\begin{array}{l}\text { Imaging } \\
\text { modality } \\
\text { Study }\end{array}$} & \multicolumn{3}{|c|}{ Optical coherence tomography } & \multirow{2}{*}{$\begin{array}{c}\begin{array}{c}\text { Cardiac magnetic } \\
\text { resonance }\end{array} \\
\text { Nakao et al }{ }^{14}(2018)\end{array}$} \\
\hline & Nishio et al'11 (2014) & Kita et al'12 (2020) & Sugizaki et al'13 (2020) & \\
\hline Subjects & Stable CAD and ACS & ACS & $\begin{array}{l}\text { Stable CAD and ACS, with } \\
\text { in-stent neoatherosclerosis }\end{array}$ & Proven or suspected CAD \\
\hline Therapies & $\begin{array}{c}16: 15 \text { for EPA } \\
\begin{array}{c}1.8 \mathrm{~g} / \text { day }+ \text { rosuvastatin vs. } \\
\text { rosuvastatin only }\end{array}\end{array}$ & $\begin{array}{c}31: 31: 35 \text { for EPA } 1.86 \mathrm{~g} / \text { day } \\
\text { vs. EPA } 0.93 \mathrm{~g} / \mathrm{day}+\mathrm{DHA} \\
0.75 \mathrm{mg} / \mathrm{day} \text { vs. no } \\
\mathrm{n}-3 \text { fatty acids }\end{array}$ & $\begin{array}{c}21: 21 \text { for EPA } \\
1.8 \mathrm{~g} / \text { day + rosuvastatin } \\
10 \mathrm{mg} / \text { day vs. rosuvastatin } \\
2.5 \mathrm{mg} / \text { day }\end{array}$ & $\begin{array}{c}50: 50: 50 \text { for EPA } 0.93 \mathrm{~g} / \text { day }+ \\
\text { DHA } 0.75 \mathrm{~g} / \text { day } \mathrm{vs} . \text { EPA } \\
1.86 \mathrm{~g} / \text { day }+ \text { DHA } 1.5 \mathrm{~g} / \text { day vs. } \\
\text { no } n-3 \text { fatty acids }\end{array}$ \\
\hline $\begin{array}{l}\text { Percentage of } \\
\text { statin-treated } \\
\text { patients }\end{array}$ & $0 \%$ & $53 \%$ & $61 \%$ & $100 \%$ \\
\hline $\begin{array}{l}\text { Baseline LDL-C } \\
\text { level in each } \\
\text { group, } \mathrm{mg} / \mathrm{dL}\end{array}$ & $\begin{array}{c}138.0 \text { vs. } 130.3 \\
\quad(P=0.41)\end{array}$ & $\begin{array}{c}120 \text { vs. } 118 \text { vs. } 125 \\
(P=0.953)\end{array}$ & $\begin{array}{c}90 \text { vs. } 89 \text { (Not shown but not } \\
\text { significant) }\end{array}$ & On-going \\
\hline Type of statins & Rosuvastatin & Rosuvastatin & Rosuvastatin & On-going \\
\hline $\begin{array}{l}\text { On-treatment } \\
\text { LDL-C in each } \\
\text { group, mg/dL }\end{array}$ & $\begin{array}{l}80.1 \text { vs. } 83.2 \\
\quad(P=0.58)\end{array}$ & $\begin{array}{l}78 \text { vs. } 82 \text { vs. } 78 \\
\text { (Not evaluated) }\end{array}$ & 68 vs. $82(P<0.001)$ & On-going \\
\hline $\begin{array}{l}\text { Primary } \\
\text { outcome }\end{array}$ & $\begin{array}{l}\text { Morphologic changes of } \\
\text { TCFAs at } 9 \text { months }\end{array}$ & $\begin{array}{l}\text { Change in minimum } \\
\text { fibrous-cap thickness } \\
\text { at } 8 \text { months }\end{array}$ & $\begin{array}{l}\text { Changes in the lipid index or } \\
\text { macrophage grade of native } \\
\text { coronary plaques at } 12 \text { months }\end{array}$ & $\begin{array}{c}\text { Change in } \\
\text { plaque-to-myocardium } \\
\text { signal intensity ratio }\end{array}$ \\
\hline Findings & $\begin{array}{l}\text { EPA+statin group increased } \\
\text { fibrous-cap thickness }(+54.8 \mu \mathrm{m} \text { vs. } \\
+23.5 \mu \mathrm{m}, \mathrm{P}<0.001) \text { and decreased } \\
\text { lipid arc }(-34.4 \text { degrees vs. }-12.7 \\
\text { degrees, } \mathrm{P}=0.007) \text { and lipid length } \\
(-2.81 \mathrm{~mm} \text { vs. }-1.2 \mathrm{~mm}, \mathrm{P}=0.009)\end{array}$ & $\begin{array}{l}\text { Primary endpoint not } \\
\text { significantly different } \\
\text { between groups (absolute } \\
\text { change: } 60 \mu \mathrm{m} \text { vs. } 20 \mu \mathrm{m} \text { vs. } \\
20 \mu \mathrm{m}, \mathrm{P}=0.1491 \text { and } \\
\text { percent change: } 58.3 \% \text { vs. } \\
14.3 \% \text { vs. } 20.0 \%, \mathrm{P}=0.1075 \text { ) }\end{array}$ & $\begin{array}{l}\text { EPA+statin group decreased } \\
\text { lipid index }(-112 \text { vs. } 29, \\
P<0.001) \text { and macrophage } \\
\text { grade }(-17 \text { vs. } 1, P<0.001)\end{array}$ & On-going \\
\hline
\end{tabular}

ACS, acute coronary syndrome; CAD, coronary artery disease; DHA, docosahexaenoic acid; EPA, eicosapentaenoic acid; LDL-C, low-density lipoprotein-cholesterol. 


\section{References}

1. Yokoyama M, Origasa H, Matsuzaki M, Matsuzawa Y, Saito Y, Ishikawa $\mathrm{Y}$, et al. Effects of eicosapentaenoic acid on major coronary events in hypercholesterolaemic patients (JELIS): A randomised open-label, blinded endpoint analysis. Lancet 2007; 369: $1090-1098$.

2. Bhatt DL, Steg PG, Miller M, Brinton EA, Jacobson TA, Ketchum $\mathrm{SB}$, et al. Cardiovascular risk reduction with icosapent ethyl for hypertriglyceridemia. N Engl J Med 2019; 380: 11-22.

3. Nicholls SJ, Lincoff AM, Garcia M, Bash D, Ballantyne CM, Barter PJ, et al. Effect of high-dose omega-3 fatty acids vs corn oil on major adverse cardiovascular events in patients at high cardiovascular risk: The STRENGTH randomized clinical trial. JAMA 2020; 324: 2268-2280.

4. Doi T, Langsted A, Nordestgaard BG. A possible explanation for the contrasting results of REDUCE-IT vs. STRENGTH: Cohort study mimicking trial designs. Eur Heart J, doi:10.1093/ eurheartj/ehab555.

5. Motoyama S, Nagahara Y, Sarai M, Kawai H, Miyajima K, Sato Y, et al. Effect of omega-3 fatty acids on coronary plaque morphology: A serial computed tomography angiography study. Circ J, doi:10.1253/circj.CJ-21-0615.

6. Alfaddagh A, Elajami TK, Ashfaque H, Saleh M, Bistrian BR, Welty FK. Effect of eicosapentaenoic and docosahexaenoic acids added to statin therapy on coronary artery plaque in patients with coronary artery disease: A randomized clinical trial. $\mathrm{J} \mathrm{Am}$ Heart Assoc 2017; 6: e006981.

7. Budoff MJ, Bhatt DL, Kinninger A, Lakshmanan S, Muhlestein $\mathrm{JB}$, Le VT, et al. Effect of icosapent ethyl on progression of coronary atherosclerosis in patients with elevated triglycerides on statin therapy: Final results of the EVAPORATE trial. Eur Heart J 2020; 41: 3925-3932.

8. Niki T, Wakatsuki T, Yamaguchi K, Taketani Y, Oeduka H, Kusunose K, et al. Effects of the addition of eicosapentaenoic acid to strong statin therapy on inflammatory cytokines and coronary plaque components assessed by integrated backscatter intravascular ultrasound. Circ J 2016; 80: 450-460.

9. Ahn J, Park SK, Park TS, Kim JH, Yun E, Kim SP, et al. Effect of n-3 polyunsaturated fatty acids on regression of coronary atherosclerosis in statin treated patients undergoing percutaneous coronary intervention. Korean Circ J 2016; 46: 481-489.

10. Watanabe T, Ando K, Daidoji H, Otaki Y, Sugawara S, Matsui $\mathrm{M}$, et al. A randomized controlled trial of eicosapentaenoic acid in patients with coronary heart disease on statins. J Cardiol 2017; 70: $537-544$.

11. Nishio R, Shinke T, Otake H, Nakagawa M, Nagoshi R, Inoue $\mathrm{T}$, et al. Stabilizing effect of combined eicosapentaenoic acid and statin therapy on coronary thin-cap fibroatheroma. Atherosclerosis 2014; 234: 114-119.

12. Kita Y, Watanabe M, Kamon D, Ueda T, Soeda T, Okayama S, et al. Effects of fatty acid therapy in addition to strong statin on coronary plaques in acute coronary syndrome: An optical coherence tomography study. J Am Heart Assoc 2020; 9: e015593.

13. Sugizaki Y, Otake H, Kuroda K, Kawamori H, Toba T, Nagasawa A, et al. Concomitant use of rosuvastatin and eicosapentaenoic acid significantly prevents native coronary atherosclerotic progression in patients with in-stent neoatherosclerosis. Circ J 2020; 84: $1826-1836$.

14. Nakao K, Noguchi T, Asaumi Y, Morita Y, Kanaya T, Fujino $\mathrm{M}$, et al. Effect of eicosapentaenoic acid/docosahexaenoic acid on coronary high-intensity plaques detected with non-contrast T1-weighted imaging (the AQUAMARINE EPA/DHA study): Study protocol for a randomized controlled trial. Trials 2018; 19: 12.

15. Nissen SE, Lincoff AM, Wolski K, Ballantyne CM, Kastelein JJP, Ridker PM, et al. Association between achieved $\omega-3$ fatty acid levels and major adverse cardiovascular outcomes in patients with high cardiovascular risk: A secondary analysis of the STRENGTH trial. JAMA Cardiol 2021; 6: 1-8. 